\title{
Variations in amount of TSST-I produced by clinical methicillin resistant Staphylococcus aureus (MRSA) isolates and allelic variation in accessory gene regulator (agr) locus
}

\author{
Miki Nagao*1, Akira Okamoto², Keiko Yamada², Tadao Hasegawa², \\ Yoshinori Hasegawa ${ }^{3}$ and Michio Ohta ${ }^{2}$
}

\begin{abstract}
Address: ${ }^{1}$ Department of Infection Control and Prevention, Kyoto University Hospital, Kyoto, Japan, ${ }^{2}$ Department of Molecular Bacteriology, Nagoya City University Graduate School of Medical Science, Nagoya, Japan and ${ }^{3}$ Department of Respiratory Medicine, Nagoya University Graduate School of Medicine, Nagoya, Japan

Email: Miki Nagao* - mnagao@kuhp.kyoto-u.ac.jp; Akira Okamoto - yoh@med.nagoya-u.ac.jp; Keiko Yamada - yakeiko@med.nagoya-u.ac.jp; Tadao Hasegawa - tadaoh@med.nagoya-c-u.ac.jp; Yoshinori Hasegawa - yhasegawa@med.nagoya-u.ac.jp; Michio Ohta - mohta@med.nagoyau.ac.jp

* Corresponding author
\end{abstract}

Published: 10 March 2009

BMC Microbiology 2009, 9:52 doi:10.1186/147I-2180-9-52
Received: I November 2008

Accepted: 10 March 2009

This article is available from: http://www.biomedcentral.com/l47I-2/80/9/52

(c) 2009 Nagao et al; licensee BioMed Central Ltd.

This is an Open Access article distributed under the terms of the Creative Commons Attribution License (http://creativecommons.org/licenses/by/2.0), which permits unrestricted use, distribution, and reproduction in any medium, provided the original work is properly cited.

\begin{abstract}
Background: Staphylococcus aureus (S. aureus) is an important pathogen associated with both nosocomial and community-acquired infections and its pathogenicity is attributed to its potential to produce virulence factors. Since the amount of toxin produced is related to virulence, evaluating toxin production should be useful for controlling $\mathrm{S}$. aureus infection. We previously found that some strains produce relatively large amounts of TSST-I; however, no reports have described the amount of TSST-I produced by clinical isolates.
\end{abstract}

Methods: Amounts of TSST-I produced by clinical methicillin resistant S. aureus (MRSA) isolates were measured by Western blotting. We determined their accessory gene regulator (agr) class by PCR and investigated whether TSST-I production correlates with variations in the class and structure of the agr.

Results: We found that $75 \%$ of surveyed MRSA isolates $(n=152)$ possessed the tst gene and that $96.7 \%$ belonged to agr class 2 . The concentrations of TSST-I secreted into culture supernatants by 34 strains measured by Western blotting differed I70-fold. Sequencing the entire agr locus $(n=9)$ revealed that some had allelic variations regardless of the amount of TSST-I produced whereas sequencing the sar, sigma factor $B$ and the tst promoter region revealed no significant changes.

Conclusion: The amounts of TSST-I produced by clinical MRSA isolates varied. The present results suggest that TSST-I production is not directly associated with the agr structure, but is instead controlled by unknown transcriptional/translational regulatory systems, or synthesized by multiple regulatory mechanisms that are interlinked in a complex manner. 


\section{Background}

Staphylococcus aureus (S. aureus) is responsible for many nosocomial and community-acquired infections. Its pathogenicity is attributed to its ability to produce many membrane-associated components and extracellular substances, several of which have been implicated as virulence factors $[1,2]$. One of the most unique manifestations among the various staphylococcal infections is staphylococcal toxic shock syndrome (TSS). The associated toxin TSS toxin-1 (TSST-1) is encoded by the tst gene, and might also be involved in the genesis of some autoimmune diseases $[1,3,4]$. The accessory gene regulator (agr) operon among several potentially associated factors is thought to positively regulate TSST-1 production [2,3]. The agr locus comprises 5 genes (AgrA, AgrB, AgrC, AgrD, and hld) that function in both transcription and translation to regulate numerous toxins, enzymes and cell surface proteins. A polymorphism in a variable region of the agr locus comprises nucleotide sequences encoding $\mathrm{AgrD}$, the C-terminal two-thirds of $\mathrm{AgrB}$, and a portion of the N-terminal half of AgrC, which has led to the assignation of $S$. aureus isolates into four classes $[2,5]$. In addition to the agr polymorphism, mutations of wild-type $S$. aureus strains resulting in agr deletions alter exoprotein biosynthesis [6]. However, the relationship between the agr polymorphism and TSST-1 production is unknown.

We previously analyzed images from two-dimensional electrophoresis (2-DE) and found that two clinical methicillin-resistant $S$. aureus (MRSA) isolates produce relatively large amounts of superantigenic exotoxins [7]. Since the amount of toxins produced is probably directly related to the virulence of $S$. aureus, evaluating the concentration of toxins produced by each strain might be useful for controlling infection.

The aim of this study was to determine whether TSST-1 production varies among clinical MRSA strains and whether it is related to variations in agr class and structure.

\section{Results}

Detection of the tst gene and agr classes

We detected the tst gene in $115(75.7 \%)$ of 152 strains after PCR amplification. Among them, 53 of 66 strains from the nation-wide collection $(80.3 \%)$ and 62 isolated from 86 blood samples $(72.0 \%)$ harbored the gene. We identified 147 of 152 isolates (96.7\%) as agr class 2, and 3 isolates as agr class $1(1.9 \%)$. We did not identify any isolates of agr classes 3 or 4 . The classes of 2 strains were unidentifiable. Among 112 tst-positive strains, 111 belonged to agr class 2 . These results indicated the clonal dissemination of a specific group of tst-positive and agr class 2 MRSA in Japanese hospitals.

\section{Evaluation of TSST-I production}

We measured the amount of TSST-1 produced in 34 randomly selected strains. The densities of the bands detected by Western blotting correlated in a semi-log manner with the amount of rTSST- 1 produced. The amounts of TSST- 1 secreted into culture supernatants evaluated by comparison with the standard curve ranged from 0.8 to $14.0 \mu \mathrm{g} /$ $\mathrm{ml}$. Thus, the amount of TSST-1 produced varied 170 -fold among clinical MRSA isolates that were cultured under the same conditions.

\section{Sequencing of the agr operon}

To determine how the structure of the agr locus influences the amount of TSST- 1 secretion, we sequenced this region in strains $1,2,3,7,8,9,10,11$ and 16, which generated a TSST-1 concentration range of 0.8 to $14.0 \mu \mathrm{g} / \mathrm{ml}$ (Table 1).

A comparison of the nucleotide sequences from the 9 strains with the corresponding sequence of the agr class 2 reference strain S. aureus SA502A (GenBank accession no., AF001782), revealed no relevant changes in the agrD and agr $\mathrm{B}$ regions, whereas 4 strains had allelic variations in the coding region of $a g r \mathrm{C}$, which is the receptor for two component regulatory systems. Strain 3 had a point mutation at nucleotide position 28 of the coding region that replaced phenylalanine with isoleucine. Strain 10 also had a point mutation at nucleotide position 651 of the coding region that replaced glutamine with histidine. Strain 8 had a 9-nucleotide deletion (nt 495 to 504 of the $\operatorname{agr} \mathrm{C}$ coding sequence) that resulted in the deletion of leucine, lysine and isoleucine. Strain 2 had a nucleotide insertion that caused a frame-shift mutation, which in turn generated numerous stop codons. Although both strains 10 and 2 produced large amounts of TSST-1, the agr locus did not consistently vary in any way from that of the other strains (Table 2). We also sequenced the pro-

Table I: Production of TSST-I evaluated by Western blotting.

\begin{tabular}{|c|c|c|c|c|c|}
\hline No. & Strain & $\mu \mathrm{g} / \mathrm{ml}$ & No. & Strain & $\mu \mathrm{g} / \mathrm{ml}$ \\
\hline I & N3I 5 & $3.5 \pm 0.22$ & 18 & 2680 & $1.4 \pm 0.19$ \\
\hline 2 & A36 & $\mid 4 \pm 1.01$ & 19 & 2681 & $1.3 \pm 0.05$ \\
\hline 3 & 3429 & $5 \pm 0.12$ & 20 & 2682 & $1.0 \pm 0.25$ \\
\hline 4 & 3472 & $|.3 \pm 0.3|$ & 21 & 2683 & $1.0 \pm 0.01$ \\
\hline 5 & 3337 & $1.1 \pm 0.20$ & 22 & 2684 & $0.8 \pm 0.02$ \\
\hline 6 & 1785 & $1.2 \pm 0.02$ & 23 & 2685 & $1.6 \pm 0.23$ \\
\hline 7 & 2271 & $2.0 \pm 0.03$ & 24 & 2686 & $2.0 \pm 0.18$ \\
\hline 8 & 3281 & $4.0 \pm 0.22$ & 25 & 2687 & $1.6 \pm 0.22$ \\
\hline 9 & 2932 & $2.8 \pm 0.19$ & 26 & 2688 & $7.6 \pm 0.07$ \\
\hline 10 & 3543 & $|4 \pm 1.2|$ & 27 & 2689 & $9.8 \pm 0.28$ \\
\hline I I & 3573 & $12 \pm 0.20$ & 28 & 2690 & $3.1 \pm 0.16$ \\
\hline 12 & V432 & $7.0 \pm 0.25$ & 29 & 2701 & $5.0 \pm 1.12$ \\
\hline 13 & V637 & $7.6 \pm 0.30$ & 30 & 2702 & $2.8 \pm 0.23$ \\
\hline 14 & V666 & $5.2 \pm 0.11$ & 31 & 2165 & $4.0 \pm 0.13$ \\
\hline 15 & V700 & $8.0 \pm 0.21$ & 32 & 3624 & $1.0 \pm 0.19$ \\
\hline 16 & V723 & $1.3 \pm 0.34$ & 33 & 3878 & $2.2 \pm 0.20$ \\
\hline 17 & V694 & $4.0 \pm 0.22$ & 34 & 3890 & $6.4 \pm 0.08$ \\
\hline
\end{tabular}

Strain I, genome strain. Strains 2 to 34 were randomly selected clinical MRSA isolates that were all tst-positive and assigned to agr class 2 by PCR. Amounts of TSST-I varied among strains and ranged from 0.8 to $14 \mu \mathrm{g} / \mathrm{ml}$. 
Table 2: Summary of nucleotide changes and predicted outcomes of mutations in the agr locus.

\begin{tabular}{|c|c|c|c|c|c|c|}
\hline Strain number & $\begin{array}{l}\text { Amount of TSST-I } \\
\text { produced }(\mu \mathrm{g} / \mathrm{ml})\end{array}$ & $\begin{array}{c}\text { Changes in agrC region } \\
\text { nucleotide sequence }\end{array}$ & Predicted outcome & tst promoter & sarA & sigB \\
\hline $\mathbf{I}$ & 3.5 & & $\mathrm{NC}$ & NC & $\mathrm{NC}$ & NC \\
\hline 2 & 14 & $T(32 I)$ insertion & Frameshift $\rightarrow$ Truncated AgrC & NC & NC & NC \\
\hline 3 & 5 & T $28 I A$ & phe $\rightarrow$ ile & NC & $\mathrm{NC}$ & $\mathrm{NC}$ \\
\hline 7 & 2 & & NC & NC & NC & NC \\
\hline 8 & 4 & $\Delta 495 \sim 504$ & Deletion of leu-lys-ile & NC & NC & NC \\
\hline 9 & 2.8 & & NC & NC & NC & NC \\
\hline 10 & 14 & G65IT & glu $\rightarrow$ his & NC & NC & NC \\
\hline I I & 12 & & NC & NC & NC & NC \\
\hline 16 & 1.3 & & NC & NC & NC & NC \\
\hline
\end{tabular}

Data are from DNA sequencing of agr loci, tst promoter region, sarA and sigB from 9 strains. All mutations were found in agrC. NC, no change.

moter regions of the tst gene, sar (staphylococcal accessory regulator) and the entire region of sigma factor $\mathrm{B}$ of these 9 strains. The sar is another positive regulatory locus for TSST-1 production that is required for maximal agr expression and sigma factor B is an important factor that feeds into the global regulatory network governing the expression of accessory genes [2,8-10]. No relevant nucleotide changes were evident in the sequences of both promoter regions of the tst gene and sar as well as the entire sigma factor $\mathrm{B}$ region (Table 2 ).

\section{Discussion}

The proportion of tst-positive isolates among clinical MRSA isolates varies from $<20 \%$ to $90 \%$ according to country and clinical background $[11,12]$. The present study found that over $75 \%$ of clinical MRSA isolates carried the tst gene. This ratio is compatible with that of recent reports from Japan and it is obviously higher than those of other countries $[11,12]$. The ratio of tst-positive isolates is increasing annually and thus it is important to understand how TSST-1 production is regulated.

The mere presence of a toxin gene does not mean that the protein will be expressed and if it is, toxin levels could widely from strain to strain. In fact, the quantity of Panton-Valentine Leukocidin (PVL) produced in vitro varies up to 10-fold among MRSA strains [13].

In the present study, we identified a 170-fold difference in the amount of TSST-1 produced among MRSA isolates by Western blotting. Expression of the tst gene is activated by agr so we sequenced the agr locus of various TSST-1 producers to determine whether it is associated with variations in TSST-1 production. Allelic variations in the agrC region were identified irrespective of the amount of TSST1 produced. One producer of a relatively large amount of TSST-1 had an insertion of nucleotides in the agrC that resulted in a frameshift, which in turn generated many stop codons. Other strains had allelic variations that resulted in replacement of an amino acid irrespective of the amount of TSST- 1 and a frameshift in the agrC of a high producer was predicted to generate truncated AgrC. Therefore, the agr locus is probably not functional with respect to TSST-1 production in those strains. Recent findings have shown that about 25\% of 105 human isolates are deficient in the production of delta-toxin, indicating that agr mediated regulation is disrupted $[14,15]$. These facts imply that mechanisms other than the agr locus are involved in TSST-1 production in our isolates. We also tried to evaluate tst gene expression by Northern blotting, but the results were not reproducible, perhaps because of high levels of expression or difficulty in removing nuclease contamination. In addition, the sequences of both the promoter region of the tst gene and the entire sar locus were conserved among these strains, indicating that these regions are not associated with variations in the amount of TSST-1 production.

The previous and present results indicate that unknown transcriptional/translational regulatory systems control TSST-1 production or that multiple regulatory mechanisms are linked in a complex manner to synthesize and produce toxin. Moreover, secretion mechanisms and proteolytic degradation would also be involved in the amount of TSST-1 produced.

A recent study has shown that variation in the amount of extracellular PVL does not correlate with the severity of infection [13]. In addition, Pragman and Schlievert noted that the transcriptional analysis of virulence regulators in animal models in vivo or in human infection do not correlate with transcriptional analysis accomplished in vitro [16]. From these viewpoints, further investigation is required to determine whether different amounts of TSST1 are produced in vivo and if so, whether they are related to clinical symptoms of diseases.

\section{Conclusion}

The present results suggest that TSST-1 production is not directly associated with the agr structure, but is instead 
controlled by unknown transcriptional/translational regulatory systems, or synthesized by multiple regulatory mechanisms that are interlinked in a complex manner.

\section{Methods \\ Bacterial strains}

Of 152 clinical MRSA isolates that we analyzed, 66 were randomly selected from the nationwide MRSA collection representing various regions of Japan in 2003, and the remainder was isolated from the bloodstream of patients in different wards at a university hospital between 1996 and 2003.

\section{Detection of the tst gene and agr-genotyping by PCR}

Bacterial chromosomal DNA was extracted after overnight growth on Luria Bertani agar as described [17]. We detected the tst gene by PCR amplification using the specific primers, TGT AGA TCT ACA AAC GAT AAT ATA AAG GAT (forward) and ATT AAG CTT AAT TAA TTT CTG CTT CTA TAG TT (reverse). Genes were amplified by denaturation for $5 \mathrm{~min}$ at $94^{\circ} \mathrm{C}$ followed by 30 cycles of $30 \mathrm{~s}$ at $94^{\circ} \mathrm{C}, 30 \mathrm{~s}$ at $52^{\circ} \mathrm{C}, 60 \mathrm{~s}$ at $72^{\circ} \mathrm{C}$ and a final extension at $72^{\circ} \mathrm{C}$ for $5 \mathrm{~min}$ in a $25-\mu \mathrm{l}$ mixture, comprising $1 \mu \mathrm{l} \mathrm{tem}-$

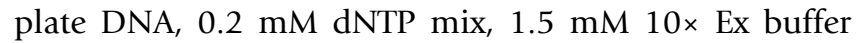
(Takara, Tokyo, Japan), 1.25 U Ex Taq (Takara) and 0.5 $\mu \mathrm{M}$ each of the forward and reverse primers. The agr class was determined by PCR amplification of the hypervariable domain of the agr locus using specific oligonucleotide primers as described [18].

\section{Preparation of recombinant partial TSST-I and anti TSST- I antibody}

Fragments of the tst gene DNA were amplified by PCR using primers with BglII-HindIII restriction sites (Table 3 ). Amplified 280-bp DNA fragments were subcloned into the pBluescriptII plasmid, digested with EcoRV and transformed into Escherichia coli DH5a, which was then digested with BglII and HindIII. The BglII -HindIII fragment of E. coli DH5 $\alpha$ was subcloned into the BamHI-Hin$d$ III site of pQE30 (Qiagen, Hilden, Germany) and transformed into E. coli JM109. His-tagged recombinant partial TSST-1 protein (rTSST-1) was expressed in E. coli JM109 and the cells were lysed using a French press (SLM Instruments, Inc., IL, USA). Recombinant TSST-1 was purified from the cell lysate using Ni-NTA agarose (Qiagen) according to the manufacturer's instructions. Purified rTSST-1 $(100 \mu \mathrm{g} / \mathrm{ml})$ was emulsified with an equal volume of Freund's complete adjuvant (Difco, NJ, USA) and subcutaneously injected into Japanese white rabbits to generate anti-TSST-1 antiserum. A second antibody response was elicited by immunization with the antigen alone and serum was collected.

\section{Quantitation of TSST-I by Western blotting}

Strains were incubated in $3 \mathrm{ml} \mathrm{LB}$ broth overnight and then $100 \mu \mathrm{l}$ cultures were transferred into $5 \mathrm{ml}$ fresh LB broth and incubated at $37^{\circ} \mathrm{C}$ with rotary shaking at 150 $\mathrm{rpm}$. Bacterial growth was monitored until the cell density reached the early stationary phase. Culture supernatant was obtained by centrifugation at $8000 \times \mathrm{g}$ for $15 \mathrm{~min}$ to precipitate bacterial cells. Total exoproteins precipitated from the culture supernatant with $10 \%$ trichloroacetic acid (TCA) were washed with cold acetone and dissolved in $100 \mu \mathrm{l}$ of Laemmli sample buffer [19]. Proteins were resolved by electrophoresis and then Western blotted according to standard procedures with the minor modification described by Whiting et al [20]. Serially diluted rTSST-1 samples were western blotted to produce a standard curve. The individual experiments to determine TSST1 expression for each strain were repeated three times. The density of each immunostained band was evaluated using Imagemaster 1D Elite ver.3.00 (Amersham Bioscience, Tokyo, Japan) and mean values were adopted.

\section{Sequence analysis of a variant agr locus}

Table 1 lists the specific primers used to sequence the entire region of agr A, B, C, and D. The region was amplified by PCR under the same conditions as described for detection of the tst gene. The products were purified using a QIAquick PCR purification kit (Qiagen) and sequenced on a CEQ 2000 DNA analysis system (Beckman Coulter, Fullerton, CA, USA) using Beckman Dye terminator cycle sequencing kits (CEQ DTCS kit, Tokyo, Japan) according to the manufacturer's instructions.

\section{Authors' contributions}

MN carried out the molecular genetic studies, participated in the sequence alignment, performed the immunoassays

Table 3: Primers used in this study.

\begin{tabular}{clll}
\hline Primer & \multicolumn{1}{c}{ Sequence $\left(5^{\prime}-3^{\prime}\right)$} & Primer & Sequence $\left(5^{\prime}-3^{\prime}\right)$ \\
\hline $2-1$ & AAA AAG CCA GCT ATA CAG TG & $2-8$ & AGT GAG GAG AGT GGT GTA AAA \\
$2-2$ & CTG AAT TAC TGC CAC GTT CT & $2-9$ & TCC GTT GTT ATT TAT GCA CCT \\
$2-3$ & CGA AT TCC ATA GGC TTT TC & $2-10$ & AGA AAG GTG TGT AGC ATA TGG \\
$2-4$ & GCC TTT TAT CTC ACG TCG TT & $2-11$ & TCC TGC AAT ACT CTT ACC AT \\
$2-5$ & TTC TTA CCA AATATG TCG CC & $2-12$ & CGA GAA TCT TAA AGT ACG TGA \\
$2-6$ & AAA AGT GGC CAT AGC TAA GT & $2-13$ & CGA AGA CGA TCC AAA ACA AA \\
$2-7$ & AGG TGC ATA AAT AAC ACG G & $2-14$ & GAT TGA ATT TGA ACG TGG AG \\
tst forward & TGT AGA TCT ACA GAT TTT ACC CCT GTT & tst reverse & ATT AAG CTT CGC TAG TAT GTT GGC TTT \\
\hline
\end{tabular}


and drafted the manuscript. KY prepared the anti TSST-1 antibody. AO participated in the sequence alignment. TH participated in the design of the study. YH and MO conceived the study and participated in its design and coordination. All authors read and approved the final manuscript.

\section{Acknowledgements}

Potential conflicts of interest. None of the authors have any conflicts.

\section{References}

I. Crossley KB, Archer GL: The Staphylococci in human disease. Churchill Livingstone, United States of America; 2000.

2. Novick RP: Pathogenicity factors of Staphylococcus aureus and their regulation. In Gram-positive pathogens Edited by: Fischetti V. Washington D.C.: ASM Press; 2000:392-07.

3. Wright JD, Holland KT: The effect of cell density and specific growth rate on accessory gene regulator and toxic shock syndrome toxin-I gene expression in Staphylococcus aureus. FEMS Microbiol Lett 2003, 2 I 8:377-383.

4. McCormick JK, Yarwood JM, Schlievert PM: Toxic shock syndrome and bacterial superantigens: an update. Annu Rev Microbiol 200I, 55:77-I04.

5. Ji G, Beavis R, Novick RP: Bacterial interference caused by autoinducing peptide variants. Science 1997, 276:2027-2030.

6. Somerville GA, Beres SB, Fitzgerald JR, DeLeo FR, Cole RL, Hoff SJ, Musser JM: In vitro serial passage of Staphylococcus aureus : changes in physiology, virulence factor production, and agr nucleotide sequence. J Bacteriol 2002, I 84: I430-I437.

7. Nakano M, Kawano $Y$, Kawagishi M, Hasegawa $T$, linuma $Y$, Ohta $M$ : Two-dimensional analysis of exoproteins of methicillinresistant Staphylococcus aureus (MRSA) for possible epidemiological application. Micro Immunol 2002, 46: I I-22.

8. Blevins JS, Gillaspy AF, Rechtin TM, Hurlburt BK, Smeltzer MS: The staphylococcal accessory regulator (sar) represses transcription of the Staphylococcus aureus collagen adhesin gene (cna) in an agr-independent manner. Mol Microbiol 1999, 33:3 17-326.

9. Chan PF, Foster J: Role of SarA in virulence determinant production and environmental signal transduction in Staphylococcus aureus. J Bacteriol 1998, 180:6232-6241.

10. Bayer MG, Heinrichs JH, Cheung AL: The molecular architecture of the sar locus in Staphylococcus aureus. J Bacteriol 1996, I 78:4563-4570.

II. Becker K, Friedrich AW, Lubritz G, Weilert M, Peters G, Christo von Eiff : Prevalence of genes encoding pyrogenic toxin superantigens and exfoliative toxins among strains of Staphylococcus aureus isolated from blood and nasal specimens. J Clin Microbiol 2003, 41 : | 434- | 439.

12. Imura S: Changes in drug susceptibility and toxin genes in Staphylococcus aureus isolated from blood cultures at a university hospital. J Infect Chemother 2004, 10:8-10.

13. Hamilton SM, Bryant AE, Carrol KC, Lockary V, Ma Y, Mcindoo E, Miller LG, Perdreau-Remington F, Pullman J, Risi GF, Salmi DB, Stevens $D L$ : In vitro production of Panton-Valentine Leukocidin among strains of methicillin-resistant Staphylococcus aureus causing diverse infections. Clin Infect Dis 2007, 45: I550- 1558.

14. Strommenger B, Cuny C, Werner G, Witte W: Obvious lack of association between dynamics of epidemic methicillin-resistant Staphylococcus aureus in central Europe and agr specificitygroups. Eur J Clin Microbio Infect Di 2003, 23:15-19.

15. McCalla C, Smyth DS, Robinson DA, Steenbergen J, Luperchio AS, Moise PA, Fowler VG, Sakoulas G: Microbiological and Genotypic Analysis of Methicillin-Resistant Staphylococcus aureus Bacteremia. Antimicrob Agents Chemother 2008, 52:344I-3443.

16. Pragman AA, Schlievert PM: Virulence regulation in Staphylococcus aureus: the need for in vivo analysis of virulence factor regulation. FEMS Immunol Med Microbiol 2004, 42:I47-I54.

17. Louie L, Matsumura SO, Choi E, Louie M, Simor AE: Evaluation of three rapid methods for detection of methicillin resistance in Staphylococcus aureus. J Clin Microbiol 2000, 38:2 I 70-2I 73.

18. Gilot P, Lina G, Cochard T, Poutrel B: Analysis of the genetic variability of genes encoding the RNA III-activating components ag $r$ and TRAP in a population of Staphylococcus aureus strains isolated from cows with mastitis. / Clin Microbiol 2002, 40:4060-4067.

19. Laemmli UK: Cleavage of structural proteins during assembly of the head of bacteriophage T4. Nature 1970, 227:680-685.

20. Whiting Jl, Rostenm PM, Chow AW: Determination by Western blot (immunoblot) of seroconversions to Toxic Shock Syndrome (TSS) Toxin I and enterotoxin A, B, or C during infection with TSS- and Non- TSS-associated Staphylococcus aureus. Infect Immu 1989, 57:231-234.
Publish with Bio Med Central and every scientist can read your work free of charge

"BioMed Central will be the most significant development for disseminating the results of biomedical research in our lifetime. "

Sir Paul Nurse, Cancer Research UK

Your research papers will be:

- available free of charge to the entire biomedical community

- peer reviewed and published immediately upon acceptance

- cited in PubMed and archived on PubMed Central

- yours - you keep the copyright
BioMedcentral 